\title{
Traduction automatique dans la formation des traducteurs : une analyse expérimentale de la post-édition
}

\author{
Automatic Translation in Translators Training: \\ an Experimental Analysis of the Post-editing
}

\author{
Teresa Tomaszkiewicz \\ Université Adam Mickiewicz de Poznań \\ tomaszki@amu.edu.pl
}

\begin{abstract}
In this article the author analyzes the evolution of the role of the translator in a world dominated by new technologies and the influence of these on the training of future translators. She focuses primarily on machine translation and post-editing. The purpose is illustrated by the results of experiments carried out within the framework of a program belonging to the EMT network and whose objective is to compare the steps of rewording in target language by post-editing and by a human translation exclusively. These experiments make it possible to draw some conclusions concerning the training of students integrating machine translation and post-editing.
\end{abstract}

Keywords: automatic translation, CAT, human translation, translators training, post-editing

\section{INTRODUCTION}

Les débuts de la traductologie remontent aux années cinquante du XXe siècle. À l'époque, cette nouvelle science s'inspirait largement des découvertes de la linguistique, notamment du structuralisme. L'objet de la recherche était le transfert du sens d'un texte à un autre sur la base d'une équivalence linguistique ${ }^{1}$. Dans cette

${ }^{1}$ À ce propos, on peut se référer, par exemple, aux travaux de Mounin, de Vinay et Darbelnet, de Catford, de Nida et de Taber. Dans la bibliographie nous ne citons pas d'œuvres concretes de ces auteurs car ils appartiennent aux références classiques de la traductologie. 
conception le traducteur devait rester invisible, et personne ne réfléchissait sur ses stratégies ou ses décisions. Il devait jouer le rôle de la fameuse "vitre transparente $»^{2}$.

À partir des années soixante-dix, la sociolinguistique, la théorie de la communication et l'analyse du discours ont influencé la réflexion traductologique en mettant l'accent sur le destinataire de la traduction (théorie du skopos) et sur l'équivalence de communication ${ }^{3}$. Parmi les différents acteurs du processus, on énumère évidemment le traducteur, qui devient plus présent dans la recherche (p. ex. les écrits de Nord), mais il n'y occupe pas le point central.

Un tournant important s'est produit dès le début des années quatre-vingt-dix. La personne du traducteur, le contexte de son travail, ses opérations mentales (mesurées par les TAPs), ses décisions traductologiques et son rôle central dans la médiation internationale deviennent d'intérêt majeur pour la recherche. La traductologie puise dans les expériences de l'analyse conversationnelle, de la sémiologie, des études sur les langues et les communications, de la théorie de l'information et bien d'autres. Or, on assiste ces dernières années à la prolifération de nouvelles technologies qui « bouleversent » le fonctionnement professionnel du traducteur.

Dans cet article, nous voulons nous concentrer sur l'évolution du rôle du traducteur dans un monde dominé par les nouvelles technologies. En effet, le traducteur contemporain ne reste plus seul avec le texte à traduire et le texte d'arrivée. Évidemment, il doit toujours déterminer les relations entre ces deux textes : d'équivalence, de correspondance, de similitude, d'adaptation, tandis que son rôle consiste toujours à assurer une réception correcte du message par le public cible. Toutefois, son travail quotidien devrait à présent reposer sur tous les outils informatisés d'aide à la traduction, TA comprise. Par conséquent, il doit assumer de nouvelles tâches comme, par exemple la pré- et la post-édition, et dans plusieurs contextes devenir terminologue, adaptateur, correcteur.

Cette réalité doit se refléter dans la formation des futurs traducteurs, ce qui fera l'objet du présent article. Tout d'abord, nous allons rappeler l'évolution de la vision des compétences technologiques qu'on devrait enseigner aux futurs traducteurs, ce qui sera illustré par un exemple du programme de formation réalisé dans notre établissement. Dans la suite, nous allons nous concentrer sur la TA et les conditions de la post-édition. Finalement, nous présenterons une expérience réalisée dans le cadre de la formation dans notre université pour comparer les étapes de mise en forme d'une post-édition et d'une traduction exclusivement humaine, ce qui nous permettra de tirer des conclusions concernant la formation à la post-édition.

\footnotetext{
${ }^{2}$ Ce terme est issu du travail de G. Mounin, Les Belles infidèles. Essai sur la traduction, 1955, où il oppose deux notions : verres transparents vs verres colorés.

${ }^{3}$ Cette fois-ci, citons, par exemple, les multiples travaux de Pergnier, Delisle, Selescovitch et Lederer.
} 


\section{2. ÉVOLUTION DE LA VISION DES COMPÉTENCES TECHNOLOGIQUES DES FUTURS TRADUCTEURS}

En 2005, J.-R. Ladmiral mettait en garde le monde traductologique en ce qui concerne l'évolution de la vision du poste de travail du traducteur :

[...] on notera que les dites nouvelles technologies de l'information et de la communication (NTIC) font converger, d'une part, la séquence informatique - qui va d'une sensibilisation à la traduction automatique (TA), c'est-à-dire en fait plus précisément à ce qu'il convient mieux d'appeler la traduction assistée par ordinateur (TAO), à l'utilisation du traitement de textes (TDT) et à l'utilisation des banques de données, tout cela tendant à la redéfinition de la station de travail du traducteur (voir Clas \& Safar, 1992), sans parler de tout ce qu'on met sous l'appellation de «localisation» - et, d'autre part, le complexe du "multimédias » : ces deux univers technologiques tendant à ne plus faire qu'un dans la pratique. Yves Gambier a insisté sur le fait que cela ne va pas sans conséquences quant au concept même de traduction qui, du coup, s'en trouve considérablement «élargi » et redéfini, ainsi que par là même en ce qui concerne les divers «métiers de la traduction » [...] (Ladmiral, 2005, § 14).

Dans cette étude Ladmiral insistait aussi sur le fait que tous ces changements doivent être pris en considération par les formateurs des futurs traducteurs pour qu'ils soient préparés aux défis des nouvelles technologies. Depuis cette réflexion de Ladmiral, plus d'une dizaine d'années s'est écoulée et ce postulat s'est matérialisé, entre autres, en forme de liste de compétences qu'un programme de formation des traducteurs devrait assurer. Dans un tel programme, la compétence technologique occupe une place importante et sa valeur croît au fur et à mesure. Nous pouvons le constater en comparant deux listes de compétences qui créent des points de référence pour un programme admis dans le réseau $\mathrm{EMT}^{4}$. La première, dont l'auteur principal est Y. Gambier, date de 2008 et elle a été intégrée dans le «wheel of competences » obligatoire jusqu'à 2018. La deuxième, proposée par D. Toudic et A. Krause, fait partie d'un nouveau document, admis par les membres du réseau EMT en mars 2018 et qui devrait constituer un repère significatif au moins pendant les cinq années à venir.

\subsection{Compétence technologique $2008-2018$}

Cette compétence est une des six présentées sous forme de «wheel of competences ", constituant une base pour deux sélections de programmes de formation des traducteurs aspirant à devenir membres de l'EMT. Elle a été précisée par quelques

\footnotetext{
${ }^{4}$ EMT : Master européen en traduction (voir : https://ec.europa.eu/info/resources-partners/europeanmasters-translation-emt/universities-and-programmes-emt-network_fr).
} 
sous-compétences de la manière suivante. Compétence technologique (maîtrise d'outils) :

- Savoir utiliser et intégrer divers logiciels de correction, de traduction, de terminologie, de mise en page et de recherche documentaire (par exemple : traitement de texte, correcteur orthographique et grammatical, Internet, mémoire de traduction, base de données terminologiques, logiciel de reconnaissance vocale).

- Savoir créer, gérer une base de données et un système de classement.

- Savoir s'adapter et se familiariser avec de nouveaux outils, notamment pour la traduction des contenus multimédias et audiovisuels.

- Savoir rédiger et réaliser une base de données dans différents formats et sur des supports de communication variés.

- Connaitre les possibilités et limites de la traduction automatique.

Comme nous le voyons, il y a 10 ans, on recommandait aux futurs traducteurs d'utiliser divers logiciels, de savoir créer des bases de données, de se familiariser avec de nouveaux outils pour la traduction AV et de connaître les possibilités de la TA. Aujourd'hui, l'acquisition de ces compétences technologiques est non seulement recommandée, mais devient absolument obligatoire.

\subsection{Compétence technologique à partir de 2018}

Dans le nouveau document (2018) décrivant les compétences à acquérir pendant la formation, la compétence technologique ${ }^{5}$ concerne tout le savoir et toutes les capacités d'introduire les nouvelles technologies présentes et celles de l'avenir dans sa propre activité de traduction. De plus, cette compétence embrasse aussi un savoir fondamental dans le domaine de la traduction automatique et la capacité d'utiliser son aide dans la mesure du besoin. Par conséquent, après la formation dans le cadre d'un programme EMT, un étudiant devrait être capable de réaliser ce qui suit :

- Utiliser les applications informatiques les plus pertinentes, y compris la gamme complète de logiciels de bureau, et s'adapter rapidement aux nouveaux outils et ressources informatiques.

- Utiliser efficacement les moteurs de recherche, les outils de corpus, les outils d'analyse de texte et les outils de TAO.

- Pré-traiter, traiter et gérer les fichiers et autres médias/sources dans le cadre de la traduction, par ex. fichiers vidéo et multimédia, gérer les technologies Web.

- Maîtriser les bases de la TA et son impact sur le processus de traduction.

\footnotetext{
${ }^{5}$ On peut consulter ce document à l'adresse suivante: EMT/emt_competence_fwk_2017_en web.pdf.
} 
- Évaluer la pertinence des systèmes de traduction automatique par rapport à sa propre traduction et, le cas échéant, mettre en œuvre un système de traduction automatique approprié.

- Appliquer d'autres outils de support aux technologies de la langue et de la traduction, comme un logiciel de gestion de travail.

La comparaison de ces deux listes de compétences que doit posséder un futur traducteur met en évidence l'importance croissante qui y est accordée. Ce n'est plus une potentialité, mais une nécessité incontournable.

In today's market, the use of technology by translators is no longer a luxury but a necessity if they are to meet rising market demands for the quick delivery of highquality texts in many languages (Bowker \& Corpas Pastor, 2015).

Il en résulte que tous les programmes de formation des traducteurs en Europe, membres de l'EMT, se doivent de dispenser ce type de formation, tout en l'améliorant et l'adaptant aux nouveautés sur le marché. Selon l'enquête Optimale ${ }^{6}$, plus de $90 \%$ des programmes de master européens forment les étudiants aux outils à mémoire de traduction. "Cet enseignement est donc aujourd'hui un fait établi et consensuel, partie intégrante de l'offre de formation universitaire, tant au niveau européen que national » (Frérot \& Karagouch, 2016, § 23). En plus, l'enquête Optimale confirme que $70 \%$ des établissements interrogés consacrent entre $10 \%$ et $25 \%$ du contenu global de leur programme de master aux outils et aux technologies d'aide à la traduction.

\subsection{Compétence technologique dans le programme posnanien}

Évidemment, ces outils technologiques sont différents, deviennent de plus en plus nombreux et performants. Ainsi, en planifiant une formation dans ce domaine, on ne peut pas prévoir l'introduction de toutes les nouveautés, même dans un proche avenir. Il est donc clair que principalement il vaut mieux préparer à un savoir-faire et à la capacité de s'adapter et de s'auto-former que de multiplier le nombre d'outils qu'on présente sans avoir suffisamment de temps pour en faire une utilisation pratique. Cette opinion est confirmée par nos étudiants, mais aussi suggérée par Cécile Frérot et Lionel Karagouch :

[...] les jeunes diplômés interrogés soulignent qu'une connaissance approfondie d'un nombre d'outils limité reste préférable à une approche superficielle d'un trop grand

\footnotetext{
${ }^{6}$ Cette enquête a cherché à établir une base de référence en matière de pratiques relatives à la formation aux outils et technologies d'aide à la traduction dans le cadre des programmes de master européens. Elle a été réalisée dans le cadre du projet OPTIMALE (Erasmus LLP), mené entre le 1.10.2010 et le 30.09.2013, dans lequel l'Université Adam Mickiewicz de Poznań était partenaire.
} 
nombre d'outils pour pouvoir répondre aux besoins du marché. Cette connaissance approfondie passe par la mise en place de méthodes d'enseignement adaptées, assurée $(2016, \S 28)$.

Par conséquent, dans le cadre du programme des études de maîtrise en traduction professionnelle et spécialisée, membre de l'EMT depuis 2011, nous offrons un cours intitulé : Traduction automatique (MT) intégrée à l'environnement CAT (outils de traduction assistée par ordinateur). Les objectifs de cette formation sont les suivants :

- Faire acquérir aux étudiants le savoir-faire visant à :

- travailler à partir des outils de MT (pré-édition),

- effectuer une post-édition des traductions automatiques,

- intégrer la MT et les outils CAT,

- créer des mémoires de traduction et les gérer - à l'aide de CAT,

- créer des bases terminologiques et les gérer - à l'aide de CAT,

- créer des projets de traduction et les gérer - à l'aide de CAT,

- assurer une reconnaissance optique du texte (OCR).

- Faire développer les terminologies concernées sur la base des textes soumis au traitement et à la traduction ${ }^{8}$.

Nous citons cet exemple, sans prétendre à une exhaustivité quelconque, mais pour bien placer l'expérience décrite plus bas. Ainsi, dans notre offre, nous proposons aux étudiants de faire connaissance de quelques outils et quelques activités menant à leur maniement, parmi lesquels il est prévu d'introduire aussi la postédition. Il s'agit tout d'abord de la post-édition brute qui «consiste à compléter, modifier, corriger, remanier, réviser et relire directement le texte produit à l'état brut par un moteur de traduction automatique » (Robert, 2010, § 7). Nous sommes conscients du fait que dans la vie professionnelle des traducteurs l'utilisation de la TA brute est beaucoup moins rentable que la post-édition évoluée qui prévoit l'imbrication TA-TAO et permet d'associer les éléments suivants :

- des mémoires de traduction (stockant des couples de phrases traduites par des traducteurs),

- des bases de données terminologiques (élaborées par des traducteurs-terminologues),

- des systèmes de traduction automatique avec règles (Robert, 2010, § 7).

\footnotetext{
${ }^{7}$ Nous sommes conscients du fait que l'abréviation anglaise CAT en français prend la forme : TAO (traduction assisté par ordinateur), mais dans le jargon professionnel en Pologne on se sert, même dans les textes français, de l'abréviation anglaise CAT, c'est pourquoi dans la suite nous allons utiliser ces deux abréviations parallèlement en fonction du contexte.

${ }^{8}$ Cet enseignement, dispensé dans les groupes réalisant le programme : PL-FR-EN, est assurée par Mme Monika Dzida-Błażejczak, enseignante mais aussi traductrice professionnelle. Elle est l'auteure de ce programme et de ses objectifs.
} 
Pourtant, avant de réaliser à un tel mariage ${ }^{9}$ et sans disposer, peut-être, de tous les outils nécessaires, nous aimerions au début, dans le cadre de notre formation, familiariser les étudiants avec la reconstruction correcte du sens d'un texte traduit automatiquement et avec certaines activités de post-édition, comme les corrections terminologiques, morphologiques et syntaxiques. Une telle activité fait aussi réfléchir les étudiants sur la nature des langues et des textes, car elle les mobilise à se poser la question sur les causes de la non-correspondance des formes de textes en différentes langues. En plus, elle rompt avec un mythe propagé par les différentes sources sur la possibilité du remplacement total de la traduction humaine par la traduction automatique ${ }^{10}$. Mais en même temps, elle montre comment le rôle du traducteur évolue dans la société contemporaine marquée par l'omniprésence des nouvelles technologies. Dans la suite, nous proposons de présenter une expérience réalisée avec les étudiants de la première année de traduction professionnelle, après le premier semestre d'études qui durent 2 ans, semestre dans lequel on introduit le cours présenté plus haut ${ }^{11}$.

\section{MODALITÉS DE L’EXPÉRIENCE}

Tout en reconnaissant les défis que posent les nouvelles technologies à la formation des futurs traducteurs, nous avons voulu nous concentrer uniquement sur la TA et la post-édition, pour vérifier dans quelle mesure la maîtrise de celle-ci aide, bloque ou influence la compréhension et la rédaction du texte final. Notre hypothèse était que les étudiants, épris par l'omniprésence de nouvelles technologies dans leur vie quotidienne, se fient aveuglement à leurs solutions, sans mobiliser leur créativité

\footnotetext{
${ }^{9}$ À propos de l'application d'une approche combinée entre TA et TAO, par exemple, dans le domaine de la finance, on peut consulter : Peraldi, Sandrine. « De la traduction automatique brute à la postédition professionnelle évoluée : le cas de la traduction financière ». Revue française de linguistique appliquée, 2016, vol. XXI, no. 1, 67-90.

${ }^{10}$ Les premiers à propager l'idée de la possibilité de remplacer l'homme par une machine dans l'activité de traduction sont avant tout les mass-médias et notamment des géants du Web, probablement financés par les firmes informatiques. Les articles et d'autres informations de ce types sont très nombreux. Citons à titre d'exemple un article dans Le Monde : http://www.lemonde.fr/pixels/article/2017/ 05/19/malgre-d-impressionnants-progres-la-traduction-automatique-a-encore-du-chemin-a-parcourir_51 30546_4408996.html_où François Yvon reste plus modéré concernant les nombreuses annonces des géants de l'Internet: "Je me méfie des annonces trop prometteuses. En allemand, une langue difficile à traduire, on avait avant tout cela un score d'une qualité de 25 sur 100. Maintenant on est à 30. On a gagné 5 points en un an, là où avant on considérait qu'il fallait un an de travail pour gagner un point. La différence est donc très significative, mais il y a encore du chemin à parcourir avant d'arriver à $100 »$.

${ }^{11}$ Nous avons organisé quelques expériences de ce type mais pour les besoins de cet article nous n'en citons qu'une seule.
} 
et leur réflexion approfondies. Cette hypothèse n'avait pas pour but de mettre en cause l'enseignement des nouvelles technologies aux futurs traducteurs, mais peutêtre de réviser la façon de l' enseigner. Ainsi, l'enquête que nous avons conçue devait nous permettre de suivre les étapes de mise en forme d'une post-édition par les étudiantes et de les comparer avec celles effectuées pendant une traduction uniquement humaine. La comparaison de ces deux savoir-faire devrait nous fournir des réponses concernant une amélioration éventuelle d'une formation à la postédition la plus efficace.

\subsection{Première partie : post-édition}

À la première partie de notre expérience ont pris part six étudiantes du programme EMT : formation des traducteurs professionnels et spécialisés. Ces étudiantes préparent un travail de maîtrise dans le domaine de la traductologie. La première partie de l'expérience se composait de trois étapes :

- La première consistait à faire post-éditer par les étudiantes la traduction automatique d'un texte de spécialité, effectuée par Google Translate. Nous avons choisi exprès le résumé d'un texte scientifique dans le domaine de la linguistique/terminologie ${ }^{12}$, car les étudiantes en question étant en train de préparer leur travail de maîtrise en traductologie devraient être familiarisées avec ce langage spécialisé grâce à plusieurs lectures effectuées au cours du séminaire qu'elles fréquentent. En outre, deux d'entre elles travaillent dans leurs recherches sur les problèmes ayant trait à la post-édition et à la TA. D'autre part, le choix d'un résumé a été dicté par le caractère concis de ce type de texte présentant un tout court, cohérent et fermé.

Voilà la traduction automatique du texte choisi $^{13}$ :

Analiza dyskursywna leksyksu zaproponowana w tym artykule ma na celu/ambicję, aby pokazać, jak i w jakich okolicznościach nowa seme (ekologiczna dla naszych badanych przypadków) została dodana do wirtualnego dictionnairique znaczenia słowa-słowa i co to jest konsekwencje. Odszyfrowanie i odkamienianie przedstawionych tworów leksykalnych dowodzi, że każdy leksem ma profil dyskursywno-leksykalny, który jest tworzony, formowany i utrwalany w momencie stwierdzenia, w którym sie znajduje. Zauważywszy, że głośniki świadomie lub nie udaja nieprzejrzystości ich wypowiedzi, zobaczymy, że profil dyskursywno-leksykalny pozwala lepiej zrozumieć utwory leksykalne, o których wygłaszaja przemówienia prasowe.

${ }^{12}$ Il s'agit d'un article de Ch. Martinez (2016), « Quelques remarques sur le profil lexico-discursif de collocations $\mathrm{N}+\mathrm{ADJ}$ (vert/durable) dans le discours médiatique », Roczniki Humanistyczne, t. LXIV, z. 8, 115-127, dont le résumé proposé par l'auteure même suit l'article, p. 127.

${ }^{13}$ Dans la suite, en citant les différentes versions du texte analysé, nous allons souligner les formes, les lexèmes ou les expressions mal traduits et par conséquent incompréhensibles. 
Nous avons divisé ce texte en 3 phrases et nous avons tout d'abord demandé aux étudiantes de souligner tous les lexèmes, expressions ou constructions qui les empêchaient de comprendre le contenu pour pouvoir introduire des corrections nécessaires. Grosso modo, dans tout le texte on peut récupérer à peu près 14 «moments difficiles » du point de vue de la compréhension. Il faut pourtant souligner que cette traduction de Google Translate paraissait la meilleure des trois dont nous disposions : l'une faite par eTranslate auquel nous avons accès par le service Machine Translation de l'UE ${ }^{14}$ et une autre faite par le même service mais de l'anglais au polonais qui repose déjà sur les études neuronales et entre dans the family of neural engines ${ }^{15}$.

Nous citons ces trois TA pour souligner que les systèmes automatiques sont encore relativement peu performants devant les textes en d'autres langues que l'anglais et le français et que souvent dans d'autres combinaisons, par exemple polonais-français, le produit de la TA reste incompréhensible. Ceci peut mener à certains scandales diplomatiques comme celui $\mathrm{du} 1^{\mathrm{er}}$ février 2018 , où la déclaration du premier ministre polonais, M. Morawiecki, traduite automatiquement par le service YouTube, contenait un contre-sens qui a provoqué de vives réactions internationales $^{16}$. Cet état des choses devrait rendre les enseignants vigilants. D'une part, ils sont obligés d'apprendre aux étudiants l'utilisation de nouvelles technologies mais de l'autre ils devraient créer chez ceux-ci des attitudes critiques par rapport à l'efficacité de la TA. Notre expérience se place alors dans cette deuxième perspective.

\footnotetext{
${ }^{14}$ Traduction faite par eTranslate : „Analiza proponowana w niniejszym artykule leksy-ożywiona jest celem/ma na celu pokazanie, w jaki sposób i w jakich okolicznościach stanie się nowe (ekologiczne) sprawy, ma znaczenie dla naprzemiennego naprzemiennego naprzemiennego naidowego organizmu, który jest możliwe do zastosowania, i jakie są jego konsekwencje. Odszyfrowywanie i twórczość powstałą w wyniku procesu odkodowywania pokazują, że każde leksyna leksyna odkodowywanie ma profil tlexy. Powstaje profil, który jest tworzony i ustalany jako deklaracja, w której się znajduje. W związku z tym zauważyjemy, że świadomie lub nie przemawiający za prelegentami zobaczymy swój profil, odpoczywając się do ico-disculsifa, co pozwala na lepsze zrozumienie wypowiedzi wygłosionego foluxent prasowi". Remarquons que le nombre de passages absolument faux ne permet pas de comprendre généralement ce texte.

${ }^{15}$ Et voilà la traduction de la version anglaise, précédant l'article même, au polonais : „Proponowana w tym artykule analiza leksykalnej i dyskursowej ma na celu wykazanie, w jaki sposób i w jakich okolicznościach nowotwór (ekologiczny dla naszych studiów przypadku) jest szczepiony na rzecz zalet i znaczenia, a co za tym idzie. Odszyfrowywanie danych, a obłuszanie pokazuje, że każda lekemch ma klestomijski profil, który jest stopniowo tworzony, formowany i załączany do deklaracji, w której się znajduje. Zauważyjąc, że mówcy są świadomie wypowiadanie się, a nie zmętnienie ich oświadczeń, zauważymy, że leksyna dyskursywny profil pozwala na lepsze zrozumienie dzieł, które przerastają

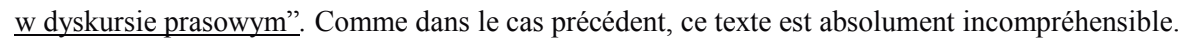

${ }^{16}$ Pour en savoir plus, on peut consulter : https://wiadomosci.wp.pl/skandaliczne-tlumaczenieoredzia-morawieckiego-google-przeprasza-6215933286398081a.
} 
Ainsi, dans cette première étape, le nombre de passages soulignés comme fautifs par chacune des étudiantes n'est pas le même et varie de 10 à 18 . Évidemment, le traitement des constructions comme fausses ou erronées semble subjectif, car dans certaines réponses les étudiantes ont souligné les passages plus longs et dans d'autres des lexèmes séparément. Or, une analyse plus détaillée montre que le manque de compréhension générale bloque la possibilité de préciser ce qui est fautif et comment remédier ponctuellement aux imperfections.

- Dans la deuxième étape, nous avons demandé aux étudiantes de proposer de nouvelles formulations, là où ceci était possible sans la connaissance de l'original, pour rendre ce texte plus intelligible. Le nombre de corrections était proportionnel au nombre de «fautes » soulignées. En somme, on a proposé de 7 à 10 solutions possibles dont, $30 \%$ étaient erronées. Nous avons aussi demandé aux étudiantes d'expliquer à l'écrit pourquoi elles n'arrivaient pas à proposer une correction plausible des passages fautifs. Les réponses à cette question se ramènent à quelques constatations répétitives :

- Manque d'un savoir spécialisé, d'une terminologie appropriée. Et pourtant, comme nous l'avons souligné plus haut, il s'agissait d'un groupe qui prépare un travail de maîtrise dans un domaine proche du sujet de l'article en question.

- Syntaxe de phrases fautive qui ne permet pas de deviner le sens.

- Traduction mot à mot ne permettant pas de reconstruire les relations à l'intérieur de toute la phrase.

- Nécessité d'avoir accès au texte original.

- Finalement, dans la troisième étape, nous avons distribué le résumé original de l'article proposé par l'auteure dans la publication mentionnée plus haut :

L'analyse lexico-discursive proposée dans cet article a pour but/ambition de montrer comment et dans quelles circonstances un sème nouveau (écologique pour nos cas étudiés) vient se greffer au sens virtuel-dictionnairique d'un mot-vocable et quelles en sont les conséquences. Le décryptage et la décortication des créations lexicales présentées prouvent que chaque lexème a un profil lexico-discursif qui se crée, se forme et se fixe au fur et à mesure de l'énoncé dans lequel il se trouve. Ayant remarqué que les locuteurs feignent sciemment ou non l'opacité de leurs énoncés, nous verrons que le profil lexicodiscursif permet une meilleure compréhension des créations lexicales dont les discours de presse fourmillent.

Cette troisième tâche consistait à reprendre la première correction et la recorriger en utilisant une autre couleur de stylo, pour que le processus de la mise en texte soit visible. Les étudiantes ont été aussi invitées à compléter le tableau de commentaires, là où ceci leur semblait important. Le résultat de toute cette expérience était tel que, malgré l'accès au texte original, aucune des étudiantes n'a été capable de présenter une version totalement satisfaisante. Pourtant des corrections acceptables ne se répartissent pas d'une manière proportionnelle. Tout au contraire, les per- 
sonnes qui au début ont pu détecter le plus grand nombre de fautes et qui ont proposé le plus de solutions acceptables, dans la dernière étape, confrontées à l'original, n'ont pas réussi à résoudre tous les problèmes. Cette observation résulte du vrai fonctionnement d'un texte scientifique dans lequel, à côté de la terminologie spécialisée, on peut trouver aussi des expressions métaphoriques, imagées ou des néologismes qui d'une part dépassent la possibilité d'une traduction automatique, mais de l'autre tout d'un coup déroutent un post-éditeur débutant. Arrêtons-nous donc sur trois exemples notoires du texte analysé :

- Le premier que nous voulons discuter embrasse une expression métaphorique : [le décryptage et] la décortification, utilisée dans un contexte de terminologie linguistique. La proposition de la traduction automatique semblait absurde : odkamienienie. Il en résulte, que dans la première correction, aucune des étudiantes n'a été capable de proposer une solution quelconque. Toutefois, l'accès au texte original a permis de suggérer les versions suivantes :

- szczegółowa analiza (la proposition qui recouvre celle d'un traducteur professionnel $^{17}$ ), mais aussi : wyszukiwanie oraz szczegółowe analizowanie;

- deux autres propositions restent encore acceptables : analiza poszczególnych elementów et dopasowanie do kontekstu;

- enfin, trois des propositions semblent absolument inappropriées : odkorkowanie; znajdowanie; oczyszczenie.

Dans ces trois derniers cas, les étudiantes expliquent l'impossibilité de comprendre et de trouver des équivalents par une méconnaissance de la terminologie spécialisée. Et pourtant le lexème utilisé dans l'original joue le rôle d'une métaphore, ce que les étudiantes n'ont pas aperçu, bloquées probablement par la proposition de la TA.

- Le deuxième point difficile est constitué par l'expression les locuteurs feignent [...] l'opacité, où le verbe feindre semble poser poser des difficultés de compréhension augmentée par la proposition de la traduction automatique : glośniki świadomie lub nie udają nieprzejrzystość. De nouveau, dans cette expression, la clarté du propos est bloquée tout d'abord par une plurivalence du terme : locuteur, ayant une signification concrète dans un discours spécialisé : mówiący; rozmówca; nadawca. Or, dans un dictionnaire informatisé, la première signification qui apparaît est : głośnik. Cette solution de la TA a partiellement dérouté les post-éditrices qui avaient du mal à comprendre l'expression : głośniki udają. Pourtant, dans la deuxième tentative de corriger ce passage les étudiantes proposent des solutions satisfaisantes : rozmówcy; odbiorcy; osoby zabierajace głos; mówcy. Toutefois, la traduction correcte du mot, locuteurs, ne garantit pas l'explicitation convenable de tout le contenu. La suggestion maladroite de la TA udają nieprzejrzystość a été conservée dans 4 traductions sur 6.

\footnotetext{
${ }^{17}$ Nous disposons de la traduction professionnelle du même résumé, parue avec l'article même.
} 
- Finalement, nous aimerions nous arrêter sur la proposition de la TA, o których wygłaszaja przemówienia prasowe. La première approche de ce fragment n'a permis à aucune personne de corriger cette expression absurde. Or, cette fois-ci, l'accès à l'original permet théoriquement de proposer de nouvelles formulations, sauf pour un exemple: mnoża się $w$ wypowiedziach prasowych, aucune autre n'est satisfaisante, plutôt incorrecte : jak donosi prasa; grzmia; formy wypowiadane w przemówieniach prasowych; formy, które komentowane sa podczas przemówień prasowych; mówia wypowiedzi prasowe. On peut déduire que la suggestion de la traduction automatique était telle que la majorité des post-éditrices ne pouvaient pas se libérer de cette première version, même après avoir lu l'original.

Nous sommes conscientes du fait que les conditions de cette expérience étaient relativement artificielles du fait qu'au début les post-éditrices ne disposaient pas du texte de l'original. Toutefois, cette organisation des tâches nous a permis une certaine observation de la façon de faire des post-éditrices débutantes et de détecter certaines étapes de leur raisonnement. Cette expérience, comme d'ailleurs d'autres avec des textes variés traduits par d'autres étudiants que nous n'avons pas la possibilité de citer ici, nous a mis en garde devant la croyance par les étudiants que les difficultés de compréhension et de réexpression résultent majoritairement de la méconnaissance d'une terminologie spécialisée, ce qui dans les passages analysés n'était pas vrai. En plus, nous avons pu avancer l'hypothèse que parfois les solutions proposées par la TA peuvent bloquer une compréhension juste et par conséquent la possibilité de correction, ce que nous avons voulu vérifier dans la deuxième partie de cette expérience.

\subsection{Deuxième partie : traduction humaine}

La deuxième tâche de notre expérience consistait à comparer les traductions post-éditées avec celles faites uniquement par les humains. Pour ce faire nous avons demandé à un autre groupe d'étudiantes ( 9 personnes) qui préparent, elles aussi, leur maîtrise en traductologie, d'exécuter une traduction « humaine », sans support d'une TA, du même texte. Les résultats de cette expérience ouvrent plusieurs voies de discussion.

- Tout d'abord, il faut constater que les traductions humaines, sans support de la TA, n'étaient pas non plus totalement correctes, mais dans la majorité des cas elles restent acceptables. En plus elles ont été faites dans une portion de temps inférieure : la post-édition durait entre 35 à 40 minutes et la traduction humaine entre 25 à 35 minutes.

- Le premier «point sensible», que nous avons discuté plus haut, concerne le lexème : décortification. Quatre traductrices débutantes sur neuf ont proposé une solution absolument acceptable (dogtębna) analiza. Toutefois, quatre autres ont 
laissé dans leurs traductions un terme inacceptable dans ce contexte : dekortyfikacja. Comme elles pouvaient se servir des dictionnaires, plus précisément de dictionnaires en ligne, elles ont pu constater que ce terme existe en polonais, mais il a une signification absolument inappropriée au contexte en question. Cette observation montre que l'utilisation des moyens électroniques d'aide à la traduction sous forme de bases de données ou de dictionnaires électroniques doit aussi être surveillée par les formateurs, car ces moyens sont souvent utilisés d'une manière dépourvue de réflexion critique.

- Le deuxième point que nous avons souligné précédemment concerne l'apparition dans la TA de la notion glośniki comme équivalent du lexème locuteurs, ce qui a dérouté en quelque sorte le premier groupe. Sans connaitre la suggestion de la TA, le deuxième groupe dans sa totalité a proposé des équivalents correctes : rozmówcy, mówcy, użytkownicy języka.

- La troisième difficulté du premier groupe résultait de la suggestion de la TA przemówienia prasowe comme équivalent de l'expression discours de presse. Dans ce cas, le deuxième groupe, sans être influencé par la suggestion de la TA, a proposé des solutions nettement meilleures : wypowiedzi prasowe, dyskursy prasowe, kreacje leksykalne w prasie.

- Et finalement, la TA a, en quelque sorte, omis le verbe fourmiller dans l'expression : dont les discours de presse fourmillent. Par conséquent, la majorité des étudiantes du premier groupe, même après avoir vu l'original, ont ignoré ce verbe dans la traduction finale. Il y en a deux qui dans les commentaires ont constaté qu'elles n'étaient pas capables de le comprendre. Ce problème n'a pas apparu dans le deuxième groupe qui a proposé des solutions acceptables : niezwykle liczne, (wypowiedzi prasowe) sa petne, (dyskurs prasowy) korzysta, aż się roi, mnoża się, obfituje ${ }^{18}$.

\section{CONCLUSION}

Jadis le processus de traduction se passait dans la tête du traducteur, qui était confronté aux textes de départ et d'arrivée et était censé de jouer un rôle d'intermédiaire entre les langues, les cultures et les pensées. À présent il est soutenu par les multiples instruments qu'offrent les nouvelles technologies. Cette aide devient nécessaire et incontournable, car elle augmente l'efficacité, comptée en temps et en volumes traduits. Or, cette aide peut par moment bloquer la créativité et ce fameux « premier réflexe ${ }^{19}$ dont jouissaient des traducteurs expérimentés. Notre observation rejoint en quelque sorte la conclusion d'une étude de M. Le Blanc :

${ }^{18}$ D'ailleurs ces solutions (3) ont été très proches de celle professionnelle: [...] wytworów leksykalnych, w które obfituje dyskurs prasowy.

${ }^{19}$ À ce propos on peut se référer à Tomaszkiewicz $(2006,2014)$. 
Sans nier que le respect des délais et la vitesse d'exécution sont des contraintes avec lesquels tous les traducteurs doivent composer, les participants à notre étude sont néanmoins d'avis que, trop « encadrés » et trop astreints aux outils d'aide à la traduction, les traducteurs ne sont plus à même de se consacrer à l'essentiel de leur travail, à exercer leur «sensibilité interculturelle » [...] et voient de ce fait leur rôle réduit à de simples «transcodeurs » dont le travail est au bout du compte peu valorisé (Le Blanc, 2016, p. 91).

Nos expérimentations, dont l'une a été présentée plus haut, montrent que certaines suggestions de la TA peuvent bloquer l'initiative des traducteurs inexpérimentés. C'est pourquoi la formation des traducteurs doit prendre en compte cette nouvelle perspective de la création humaine, où les relations entre le traducteur et le texte passent par l'intermédiaire des machines qui peuvent être utiles mais qui souvent devraient être corrigées elles-mêmes.

À présent, on consacre beaucoup de recherches à l'utilité/nécessité de nouvelles technologies dans le travail quotidien d'un traducteur. On cite ces différents outils et leur performativité, leur variabilité (Robert, 2013 ; Bowker \& Corpas, 2015 ; Witczak, 2016) ; on présente des conseils concernant les possibilités de leur utilisation (Bowker, 2002; Witczak, 2016), on cite beaucoup de progrès dans ce domaine (Robert, 2013 ; Peraldi, 2016). Or, rares sont des études ponctuelles qui décrivent, pas à pas, le savoir-faire des étudiants, futurs traducteurs. En plus, rarement on essaie de comparer la façon de faire d'un étudiant intégré dans un programme de traduction humaine avec celle d'un étudiant réalisant une post-édition. Comme nous avons avancé plus haut, notre étude n'avait pas pour but de mettre en cause la formation aux nouvelles technologies, mais de montrer les points faibles de celle-ci.

Ainsi, tout en reconnaissant que notre expérience ne peut pas prétendre à une exhaustivité, nous pensons qu'elle constitue une nouveauté dans le débat se rapportant à la présence de la technologie dans les programmes de formation des futurs traducteurs. La première observation qui en découle est telle, que les formateurs doivent mettre en garde les futurs traducteurs contre une croyance aveugle en une omni potentialité des solutions techniques à tous les problèmes traductologiques. La deuxième est plus large et consiste à suggérer aux auteurs des programmes de formation de les diversifier en fonction de la maturité des futurs traducteurs, en leur suggérant tout d'abord une approche intermédiaire entre la traduction humaine et la TA pour après passer à une post-édition proprement dite. 


\section{RÉFÉRENCES BIBLIOGRAPHIQUES}

Bowker, L. (2002). Computer-Aided Translation Technology. Ottawa : University of Ottawa Press.

Bowker, L. \& Corpas Pastor, G. (2015). « Translation Technology ». In R. Mitkov (réd.), Handbook of Computational Linguistics. Oxford : Oxford University Press.

Clas, A. \& Safar, H. (réd.). (1992). L'Environnement traductionnel. La station de travail du traducteur de l'an 2001. Actes du colloque de Mons, 25-27 avril 1991. Montréal : AUPELF \& Presses de l'Université du Québec, coll. «Actualité scientifique ».

Frérot, C. \& Karagouch, L. (2016). « Outils d'aide à la traduction et formation de traducteurs : vers une adéquation des contenus pédagogiques avec la réalité technologique des traducteurs ». ILCEA, 27. Mis en ligne le 08 novembre 2016. Accessible sur le site http://journals. openedition.org/ilcea/3849. Dernière consultation le 30 avril 2018.

Gambier, Y. (2000). "Le traducteur des multimédias : une nouvelle identité professionnelle ». In S. Mejri, A. Clas, T. Baccouche \& G. Gross (réd.), La traduction: théories et pratiques. Actes du colloque international "Traduction humaine, traduction automatique, interprétation », Tunis 28-30, septembre 2000. Vol. I (pp. 111-121), cité par Ladmiral (2005).

Ladmiral, J.-R. (2005). «Formation des traducteurs et traduction philosophique ». Meta, vol. 50, no. $1,96-106$.

Le Blanc, M. (2016). «La traduction spécialisée à l'ère des nouvelles technologies : Quel impact sur le texte de spécialité ?». Studia Romanica Posnaniensia, XLIII (1), 77-92.

Mounin, G. (1955). Les Belles infidèles. Essai sur la traduction. Lille : Presses Universitaires du Septentrion.

Peraldi, S. (2016). « De la traduction automatique brute à la post-édition professionnelle évoluée : le cas de la traduction financière ». Revue française de linguistique appliquée, vol. XXI, no. 1, 67-90.

Robert, A.-M. (2013). « La post-édition : l'avenir incontournable du traducteur ? ». Traduire, 222, 137-144. Mis en ligne le 12 novembre 2013. Accessible sur le site http://journals.opene dition.org/traduire/460. Dernière consultation le 03 avril 2018. DOI : 10.4000/traduire.460.

Tomaszkiewicz, T. (2006). "Traductions collaboratives en tant que moyen pour analyser les stratégies de compréhension et de mise en forme ». In S. Öztürk Kasar, Interdisciplinarité en traduction. Vol. II (pp. 81-94). Istanbul : Les éditions ISIS.

Tomaszkiewicz, T. (2014). «Traductions collaboratives : lieu de rencontre entre professionnels, chercheurs et formateurs ». Tralogy, II, session 6 : Teaching around MT/Didactique, enseignement, apprentissage, mis à jour le. Accessible sur le site http://odel.irevues.inist.fr/tralogy/ index.php?id=257. Dernière consultation le 21 mai 2014.

Tual, M. (2017). "Malgré d'impressionnants progrès, la traduction automatique a encore du chemin à parcourir ». Le Monde. Accessible sur le site http://www.lemonde.fr/pixels/article/ 2017/05/19/malgre-d-impressionnants-progres-la-traduction-automatique-a-encore-du-chemina-parcourir_5130546_4408996.html\#flf3p4kbe1113UO8.99. Dernière consultation le 19 mai 2017.

Witczak, O. (2016). « Tłumacze kontra maszyny, czyli o thumaczeniu wspomaganym komputerowo ». In B. Whyatt \& al., Thumacz - praktyczne aspekty zawodu (pp. 201-231). Poznań : Wydawnictwo Naukowe UAM. 